\title{
Article \\ Interplay between Thickness, Defects, Optical Properties, and Photoconductivity at the Centimeter Scale in Layered GaS
}

\author{
Stefano Dicorato, Yael Gutiérrez, Maria M. Giangregorio, Fabio Palumbo (D), Giuseppe V. Bianco (D) \\ and Maria Losurdo *(D)
}

check for updates

Citation: Dicorato, S.; Gutiérrez, Y.; Giangregorio, M.M.; Palumbo, F.; Bianco, G.V.; Losurdo, M. Interplay between Thickness, Defects, Optical Properties, and Photoconductivity at the Centimeter Scale in Layered GaS. Nanomaterials 2022, 12, 465. https:// doi.org/10.3390/nano12030465

Academic Editor: José

Antonio Sánchez-Gil

Received: 24 December 2021

Accepted: 26 January 2022

Published: 28 January 2022

Publisher's Note: MDPI stays neutral with regard to jurisdictional claims in published maps and institutional affiliations.

Copyright: (c) 2022 by the authors. Licensee MDPI, Basel, Switzerland. This article is an open access article distributed under the terms and conditions of the Creative Commons Attribution (CC BY) license (https:/ / creativecommons.org/licenses/by/ $4.0 /)$.
Institute of Nanotechnology, CNR-NANOTEC, c/o Dipartimento di Chimica, Università di Bari, via Orabona 4, 70126 Bari, Italy; stefano.dicorato@nanotec.cnr.it (S.D.); yael.gutierrezvela@nanotec.cnr.it (Y.G.); michelaria.giangregorio@nanotec.cnr.it (M.M.G.); fabio.palumbo@cnr.it (F.P.); giuseppevalerio.bianco@cnr.it (G.V.B.)

* Correspondence: maria.losurdo@cnr.it

\begin{abstract}
From the group-III monochalcogenide (MX, M = Ga, In; X = S, Se, Te) layered semiconductors, gallium monosulfide, GaS, has emerged as a promising material for electronics, optoelectronics, and catalysis applications. In this work, GaS samples of various thicknesses in the range from 38 to $1665 \mathrm{~nm}$ have been obtained by mechanical exfoliation to study the interplay between structural, morphological, optical, and photoresponsivity properties as a function of thickness. This interplay has been established by analyzing the structure through Raman spectroscopy and X-ray diffraction, the morphology through scanning electron microscopy and atomic force microscopy, the density and optical properties through spectroscopic ellipsometry, and the photoresponsivity through currentvoltage measurements under UV light. This work shows that photoresponsivity increases with increases in GaS thickness, resulting in a UV photoresponsivity of $1.5 \cdot 10^{-4} \mathrm{AW}^{-1}$ stable over several on/off cycles.
\end{abstract}

Keywords: gallium monosulfide; layered chalcogenide; UV-photoresponsivity; ellipsometry

\section{Introduction}

Layered group-III monochalcogenide semiconductors $\mathrm{MX}(\mathrm{M}=\mathrm{Ga}$ and $\mathrm{In}, \mathrm{X}=\mathrm{S}$, Se, and $\mathrm{Te}$ ) have emerged as potential candidates for photocatalytic [1,2] and optoelectronic applications [3,4] due to their stability, tunable bandgap, and high carrier mobility. Among those, gallium monosulfide, GaS, is a layered van der Waals (vdW) semiconductor with a hexagonal lattice, where each layer consists of S-Ga-Ga-S atoms covalently bonded and six-membered $\mathrm{Ga}_{3} \mathrm{~S}_{3}$ rings. Each S-chalcogen atom is $\mathrm{sp}^{3}$ hybridized, with three of the $\mathrm{sp}^{3}$ orbitals forming Ga-S bonds; the remaining S-chalcogenide orbital is occupied by lone-pair electrons forming vdW interactions between layers, as shown in Figure 1. GaS holds a special place in the family of layered monochalcogenides because of its wide energy bandgap, which extends up to the UV region. Specifically, GaS is a wide, indirect bandgap semiconductor with experimental bandgap values reported in the range from 2.34 to $2.62 \mathrm{eV}$ depending on the deposition technique [5-10], whereas values of $3.02 \mathrm{eV}$ [11] and $3.33 \mathrm{eV}$ [12] of the indirect bandgap have been reported for bilayer and monolayer GaS, respectively.

A few layers of $\mathrm{GaS}$ can be deposited by different methods, such as chemical vapor deposition (CVD) [13,14], transport reaction [5,9], pulsed laser deposition [10,15], and mechanical exfoliation $[3,16]$.

The recent interest in GaS stems from its exploitation in photocatalysis, electrochemical hydrogen production by water splitting [17], energy storage [18], nonlinear optics [19], gas sensing [20], and ultraviolet selective photodetectors [21,22]. Among various approaches to photodetectors, the low-dimensional semiconductor-based photodetectors are very attractive because of the relatively easy fabrication by exfoliation; nevertheless, subsequent 
mechanical exfoliation that reduces the thickness results in ripples, wrinkles, cracks, and the non-homogeneous thickness of flakes.

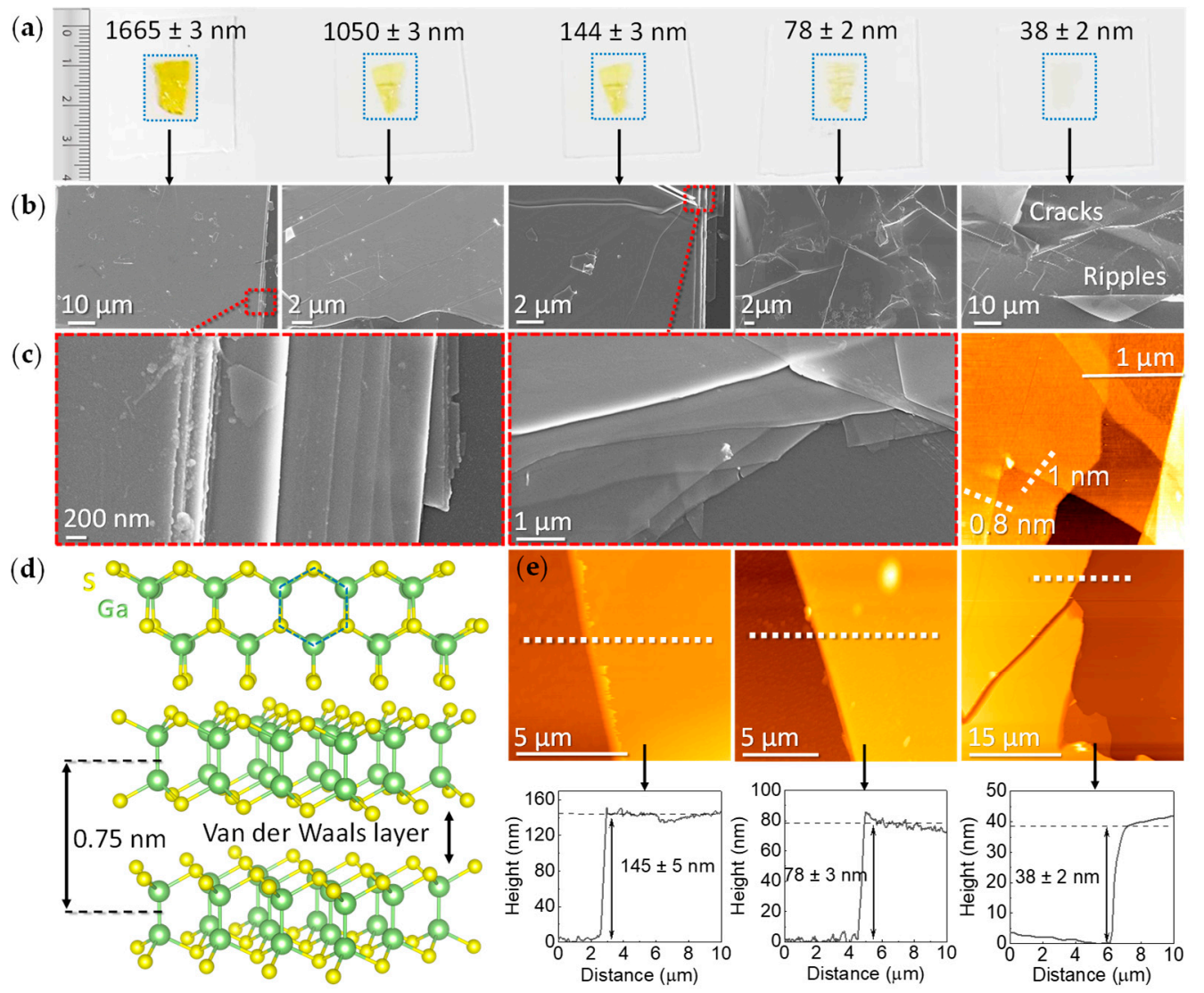

Figure 1. (a) Picture of exfoliated GaS of various thicknesses transferred onto glass. (b) Corresponding SEM images of the exfoliated GaS samples in (a) as a function of thickness. (c) Details from SEM and AFM images of the edges of samples to show the GaS layered structure. (d) Top view and side view of the GaS crystal structure, showing the monolayer composed of S-Ga-Ga-S bonds and the Van der Waals interaction between layers; the thickness of $0.75 \mathrm{~nm}$ for a monolayer is also shown. (e) AFM images and line profiles for the 144, 78, and $38 \mathrm{~nm}$ samples, from which average thickness has been determined.

In this paper, we discuss the dependence on the thickness of the interplay between the structural and optical properties and the photoresponsivity of GaS samples obtained by mechanical exfoliation. The investigated samples have a thickness greater than $30 \mathrm{~nm}$ (which corresponds to approximately $30 \mathrm{GaS}$ layers); this has been verified using optical microscopy, which shows that continuous flakes on a centimeter scale can be obtained for this thickness. The mechanically exfoliated GaS layers have been transferred onto glass substrates and characterized structurally using Raman spectroscopy and X-ray diffraction (XRD), morphologically by scanning electron microscopy (SEM) and by atomic force microscopy (AFM), and optically using spectroscopic ellipsometry. We show that photoresponsivity decreases with the decrease in GaS thickness, which is correlated to the density, polarizability, and light absorption of the GaS layers. Finally, we investigate the applicability of the exfoliated GaS samples to UV-light detection, demonstrating good cyclability and on/off current ratios in the range from $10^{4}$ to $10^{5}$.

\section{Materials and Methods}

Grown by the Bridgman method, $c$-axis (0001)-oriented hexagonal Gallium (II) Sulfide $(\beta-\mathrm{GaS})$ crystals were purchased by 2D Semiconductor (nominal purity $>99.99 \%$ ). Mechanical exfoliation by the thermal tape release method was performed on the as-received 
crystals, with subsequent transferring onto Gorilla glass substrates of GaS flakes. By subsequent exfoliations, samples with a thickness in the range from 38 to $1665 \mathrm{~nm}$ were obtained.

Raman spectroscopy was performed using a LabRAM HR Horiba spectrometer (HORIBA Ltd., Palaiseau, France), with a $532 \mathrm{~nm}$ wavelength continuous excitation laser source. Measurements were carried out on several points of the samples in the 50-1000 $\mathrm{cm}^{-1}$ range at room temperature.

After exfoliation, the crystalline quality was checked by X-ray diffraction (XRD) in thin film geometry. The measurements were performed with an Ultima IV diffractometer (Rigaku Corp., Akishima, Japan), equipped with parallel beam optics and a thin film attachment, using $\mathrm{Cu} \mathrm{K} \alpha$ radiation $(\lambda=1.5405 \AA)$, operated at $30 \mathrm{~mA}$ and $40 \mathrm{kV}$, over the $2 \theta$ range from 5 to $70^{\circ}$, at a scanning rate of $1^{\circ} / \mathrm{min}$, with a step width of $0.02^{\circ}$.

Scanning Electron Microscopy (SEM) was performed with a Zeiss Supra 40 FEG SEM (Zeiss, Oberkochen, Germany) equipped with a Gemini field emission gun operated at an extraction voltage of $3 \mathrm{kV}$ and a $30 \mu \mathrm{m}$ aperture.

XPS measurements were carried out using a Scanning XPS Microprobe (PHI 5000 Versa Probe II, Physical Electronics, MN, USA) equipped with a monochromatic Al K $\alpha$ x-ray source $(1486.6 \mathrm{eV})$ with a spot size of $200 \mu \mathrm{m}$. Survey $(0-1200 \mathrm{eV})$ and high-resolution spectra (C 1s, O 1s, S2p, S2s, Ga2p3, Ga3d, and valence band region) were recorded in FAT mode at a pass energy of 117.40 and $29.35 \mathrm{eV}$, respectively. Spectra were acquired at a take-off angle of $45^{\circ}$ with respect to the sample surface. Surface charging was compensated using a dual- beam charge neutralization system, the hydrocarbon component of C1s spectrum was used as an internal standard for charging correction, and it was fixed at $285 \mathrm{eV}$.

Spectroscopic Ellipsometry (HORIBA Ltd., Palaiseau, France) was used to measure the room temperature spectra of the refractive index and the extinction coefficient in the 0.75-6.5 eV photon energy range with $0.05 \mathrm{eV}$ resolution. Ellipsometric spectra at the incident angle of $70^{\circ}$ were herein represented, although variable angle spectra in the range from 55 to $75^{\circ}$ were acquired to check possible anisotropy of the samples, with the crystal structure being hexagonal. Indeed, because of the mosaicity of the various flakes and defects from the exfoliation, the isotropy assumption worked well. The experimental spectra of the complex pseudodielectric function, $\langle\varepsilon\rangle=\left\langle\varepsilon_{1}\right\rangle+\mathrm{i}\left\langle\varepsilon_{2}\right\rangle$, were fitted by an ambient air/GaS + voids/glass model. The glass substrate was measured before transferring the GaS. The GaS dielectric function from a single crystal [23] was combined to voids representing the defects according to the Bruggeman effective medium approximation (BEMA) [24] under isotropic fitting assumption.

After the deposition of silver (Ag)-paste electrodes ( $~ 50 \mathrm{~nm}$ thick), placed $0.5 \mathrm{~cm}$ apart on top of the GaS layers, electrical characterization was performed using a Keithley617 Programmable Electrometer (Keithley Instruments, OH, USA), scanning voltages from -10 to $+10 \mathrm{~V}$. Photoresponse was investigated using an AM1.5 spectrum halogen $100 \mathrm{~mW} \mathrm{~cm}^{-2}$ lamp and a $405 \mathrm{~nm}$ laser $\left(250 \mathrm{~mW} \mathrm{~cm}^{-2}\right)$ as visible and UV sources, respectively.

\section{Results}

\subsection{Structural and Optical Characterization}

Figure 1 shows the centimeter scale exfoliated GaS layers of different thicknesses transferred onto glass, with the peculiar yellow color (due to the bandgap of $\approx 2.50 \mathrm{eV}$ ) vanishing with the decrease in thickness caused by a decrease in light absorption. The change in color is due to the change in thickness and not in the indirect bandgap, which stays at $2.50 \mathrm{eV}$ in the investigated range of thickness; specifically, by DFT calculations and experimental ellipsometric measurements, it was discovered that, for a number of layers higher than five (approximately $5 \mathrm{~nm}$ ), the bandgap value has a bulk value. The morphological features, obtained by scanning electron microscopy (SEM) and atomic force microscopy (AFM) for the samples of various thicknesses, clearly indicate that the layered structure of the samples with structural defects, i.e., cracks, wrinkles, ripples, and flake boundaries, increase with the number of exfoliations and, hence, with the decrease in 
the thickness. The thickness values reported in Figure 1a were obtained by the fitting of ellipsometric spectra (see the Discussion section). The reported errors result from the ellipsometric analysis on a spot size of $2 \mathrm{~mm} \times 4 \mathrm{~mm}$. These thicknesses are compared to those in Figure 1e obtained by the AFM line profiles, where the standard deviation was calculated on ten line profiles in various positions per sample. AFM topographies also show that, at the layer level, the surface is very smooth and clean, ruling out residuals from the tape transfer.

The crystalline quality and structure of the exfoliated samples were assessed by Raman scattering and X-ray diffraction. The XRD diagram of the thin $38 \mathrm{~nm}$ exfoliated sample in Figure 2a shows prominent peaks corresponding to the GaS (002) and GaS (004) reflections of the $c$-axis oriented $2 \mathrm{H}$ structure of the $\beta$-GaS hexagonal phase, with lattice constants of $a=b=0.3585 \mathrm{~nm}$ and $c=1.5500 \mathrm{~nm}$, and the (006) and (0010) reflections visible in the inset. The aforementioned are visible on all of the exfoliated samples. By increasing the number of exfoliations to reduce the thickness, additional peaks corresponding to the GaS (105) and $\mathrm{GaS}$ (107) reflections appear due to different planes exposed by the flakes' edges.

Figure $2 b$ shows the Raman spectra of the samples with different thicknesses. From the irreducible representation of the $\mathrm{D}^{4}{ }_{6 \mathrm{~h}}$ point group, GaS presents phonon modes $\mathrm{A}^{1}{ }_{1 \mathrm{~g}}$ at $187 \pm 1 \mathrm{~cm}^{-1}, \mathrm{~A}^{2}{ }_{\mathrm{g}}$ at $359 \pm 1 \mathrm{~cm}^{-1}$, and $\mathrm{E}^{1}{ }_{2 \mathrm{~g}}$ at $294 \pm 1 \mathrm{~cm}^{-1}$.

The intensity of all Raman modes decreases with the decrease in thickness; the $\mathrm{E}^{1}{ }_{2 \mathrm{~g}}$ mode becomes barely visible in the few-layer regime, which is consistent with the trends already reported in the literature $[25,26]$. The ratio $A^{1}{ }_{1 g} / A^{2}{ }_{1 g} \sim 1$, typical of the single crystals, is preserved for all exfoliated samples, indicating that the layers' stacking is unaffected by the exfoliation process. Furthermore, there is no significant shift in the frequency of both the $\mathrm{A}^{1}{ }_{1 \mathrm{~g}}$ and $\mathrm{A}^{2}{ }_{1 \mathrm{~g}}$ Raman modes; consistent with previous reports on GaS, wavenumber shifts are approximately $1 \mathrm{~cm}^{-1}$, which is within the error bar of peak position indicated. This is different from what is typically observed in other bidimensional chalcogenides, such as $\mathrm{MoS}_{2}$ [27], where the position of Raman modes can be used to identify the number of layers as the wavenumber of the $\mathrm{MoS}_{2} \mathrm{~A}_{1 \mathrm{~g}}$ mode decreases whereas that of $E_{2 g}$ increases with the decrease in thickness. The softening of the in-plane $E_{2 g}$ mode (of about $4 \mathrm{~cm}^{-1}$ from bulk to monolayer) in $\mathrm{MoS}_{2}$ with the increase in thickness is due to the decrease in the long-range Coulombic interaction between Mo atoms. In contrast, the out-of-plane $A_{1 g}$ mode wavenumber increase (approximately $5 \mathrm{~cm}^{-1}$ from monolayer to bulk) with the increase in thickness is due to restoring forces on the S-atoms due to interlayer interactions. Therefore, the negligible shift for GaS indicates a negligible interaction between the layers and negligible stress/strain in the GaS samples stemming from the exfoliation. On the contrary, as shown in Figure 2c, the full width at half maximum (FWHM) of the peaks increases with the decrease in thickness because of the larger density of defects induced by the exfoliation, which is consistent with the SEM images in Figure 1b. The increased density of wrinkles and edges also affects the oxidation of the samples as indicated by the XPS data in Figure 2e-f. Specifically, the O/Ga atomic percentage ratio increases with the exfoliation runs and with the decrease in GaS thickness. Correspondingly, the fitting of the Ga3d and S2p photoelectron core levels shows a higher contribution of $\mathrm{Ga}-\mathrm{O}$ and defective sulfur at the high binding energy side for the thin GaS sample, which is consistent with the assumption that GaS oxidation occurs preferentially at the edges and wrinkles. 
(a)

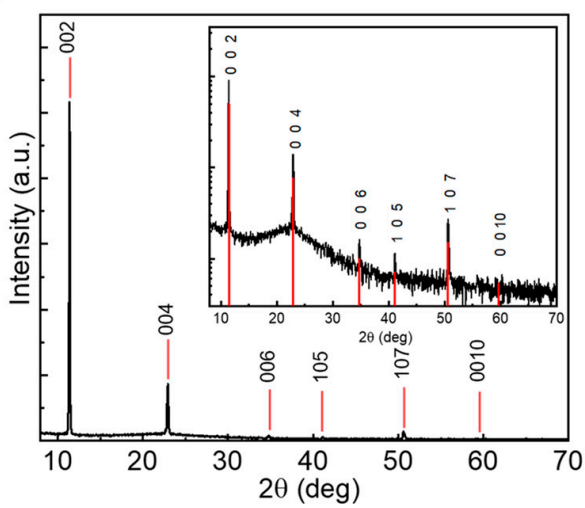

(c)

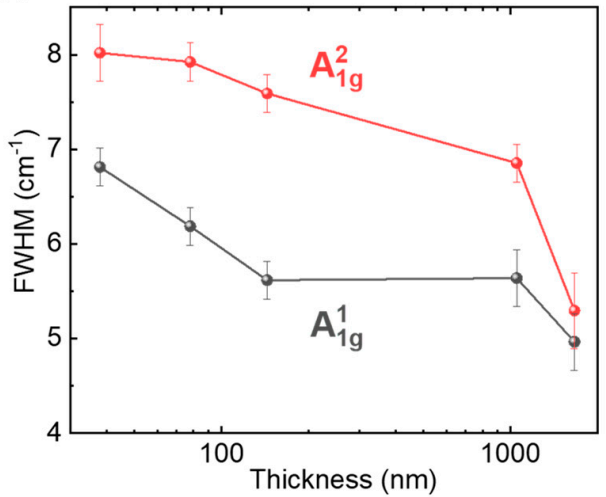

(e)

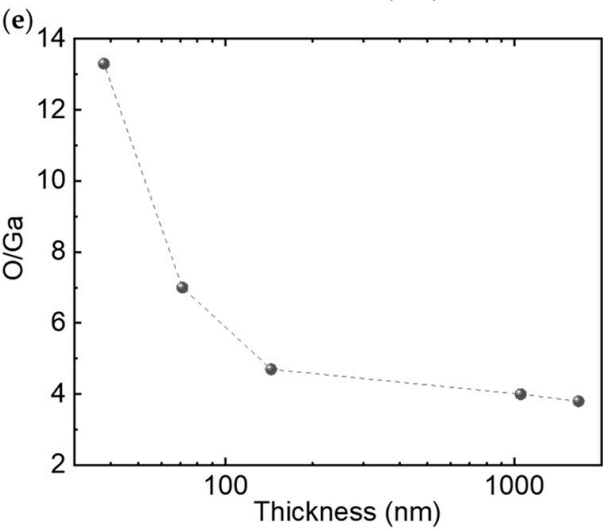

(b)

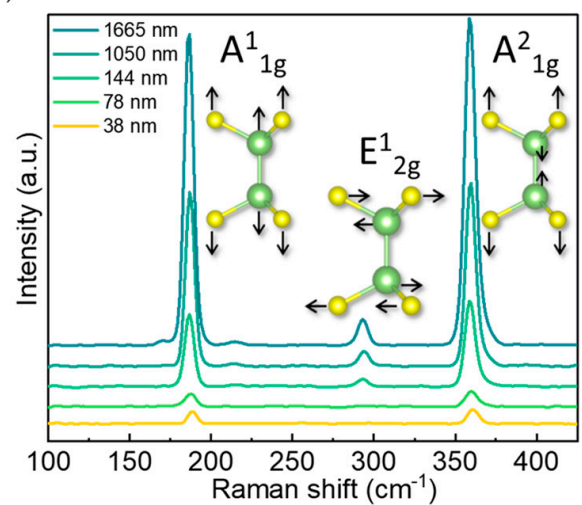

(d)

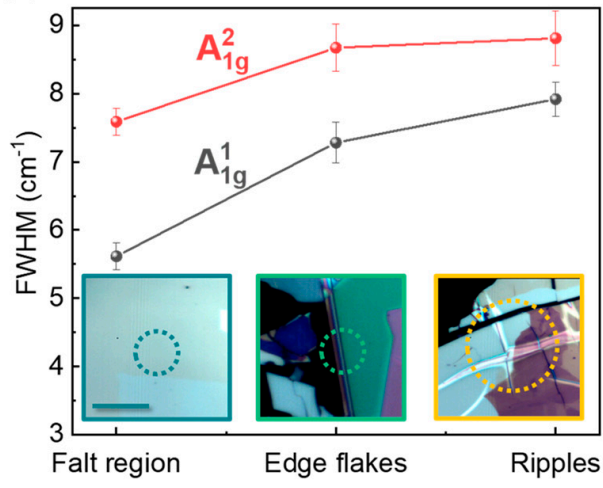

(f)
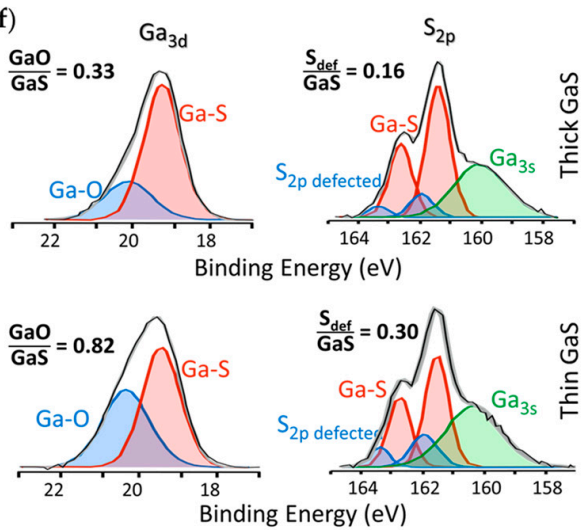

Figure 2. (a) XRD diagram of the $38 \mathrm{~nm}$ exfoliated GaS. (b) Raman spectra of exfoliated GaS with different thicknesses. (c) Dependence of the FWHM of the $\mathrm{A}^{1}{ }_{1 \mathrm{~g}}$ and $\mathrm{A}^{2}{ }_{1 \mathrm{~g}}$ modes as a function of the GaS average thickness. (d) FWHM of the $\mathrm{A}^{1}{ }_{1 \mathrm{~g}}$ and $\mathrm{A}^{2}{ }_{1 \mathrm{~g}}$ modes measured at the flat basal plane of the flake, at the edge of the flakes, and at the ripple position (as shown by the micrographs in the inset). (e) O/Ga atomic percentage ratio as determined from the Ga3d and O1s XPS peaks as a function of flakes thickness. (f) XPS spectra of the Ga3d and S2p photoelectron core levels for the thin and thick $\mathrm{GaS}$ flakes. The ratio of the Ga-oxide $(\mathrm{GaO})$ to $\mathrm{GaS}$ fitting components from the Ga3d and that of the defective sulfur to GaS from the S2p are also indicated.

The optical properties, as determined by spectroscopic ellipsometry, are shown in Figure 3. The spectra of the real, $\left\langle\varepsilon_{1}\right\rangle$, and imaginary, $\left\langle\varepsilon_{2}\right\rangle$, parts of the pseudodielectric function, $\langle\varepsilon\rangle=\left\langle\varepsilon_{1}\right\rangle+i\left\langle\varepsilon_{2}\right\rangle$, show an interference system from which the sample thickness has been derived through a fitting routine using the model sketched in the inset. For the GaS layer, we used a BEMA mixture of the GaS dielectric function from [23] and voids, which represent defects; the \% volume fraction of voids increases with the decrease in thickness consistently with the increase in defects and cracks seen via SEM. The spectra of the imaginary part of pseudodielectric function, $\left\langle\varepsilon_{2}\right\rangle$, show two main peaks at $3.95 \mathrm{eV}$ and at $5.45 \mathrm{eV}$ 
corresponding to interband critical points, with the former attributed to the transitions between the highest valence band and the lowest conduction band in the neighborhood of the high symmetry points $\mathrm{K}$ and $\mathrm{H}$ in reciprocal space where the bands run parallel and the latter assigned to electronic transition along the $\Gamma-\mathrm{M}$ and $\mathrm{A}-\mathrm{L}$ paths between the deeper valence and the conduction band. The fundamental indirect energy gap $\mathrm{E}_{0}$ is at $2.50 \mathrm{eV}$, and it does not change in the investigated range of thickness. Interestingly, the amplitude of the critical points decreases with the decrease in thickness. Good agreement was observed between the ellipsometric thickness and the AFM line profiles. Figure $3 c, d$ show, respectively, the effective refractive index (which includes the voids representing the defects) and effective extinction coefficient at $3.06 \mathrm{eV}$ (i.e., $405 \mathrm{~nm}$ corresponding to the UV light wavelength used during the photoresponsivity measurements, as shown below) as a function of the thickness of the GaS exfoliated samples. From these plots, it can be inferred that, by decreasing the GaS thickness, the effective density and light absorption of samples decreases because of the finite dimension of the flakes. Furthermore, the shadowed yellow region indicates that two regimes of thickness can be identified, namely for thicknesses below $1000 \mathrm{~nm}$, where the effective density and light absorption increases with thickness, whereas a plateau is reached above $1000 \mathrm{~nm}$; however, this is lower than the bulk GaS crystal because of the defects introduced by the exfoliation.

(a)

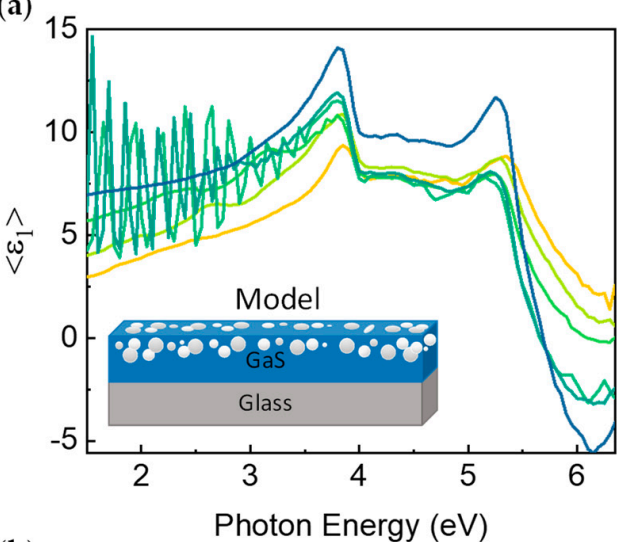

(b)

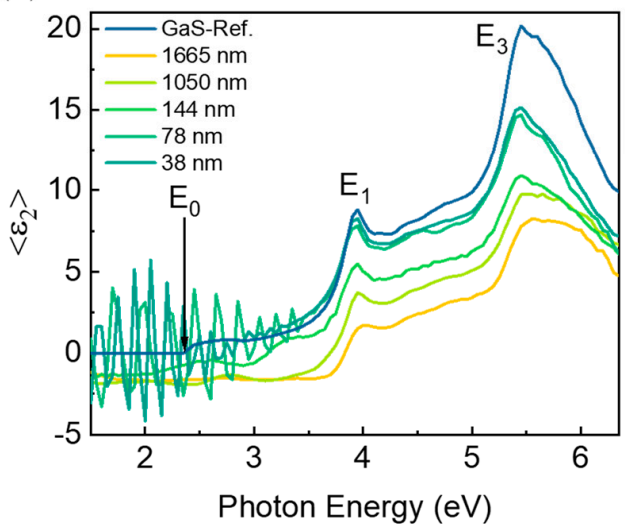

(c)
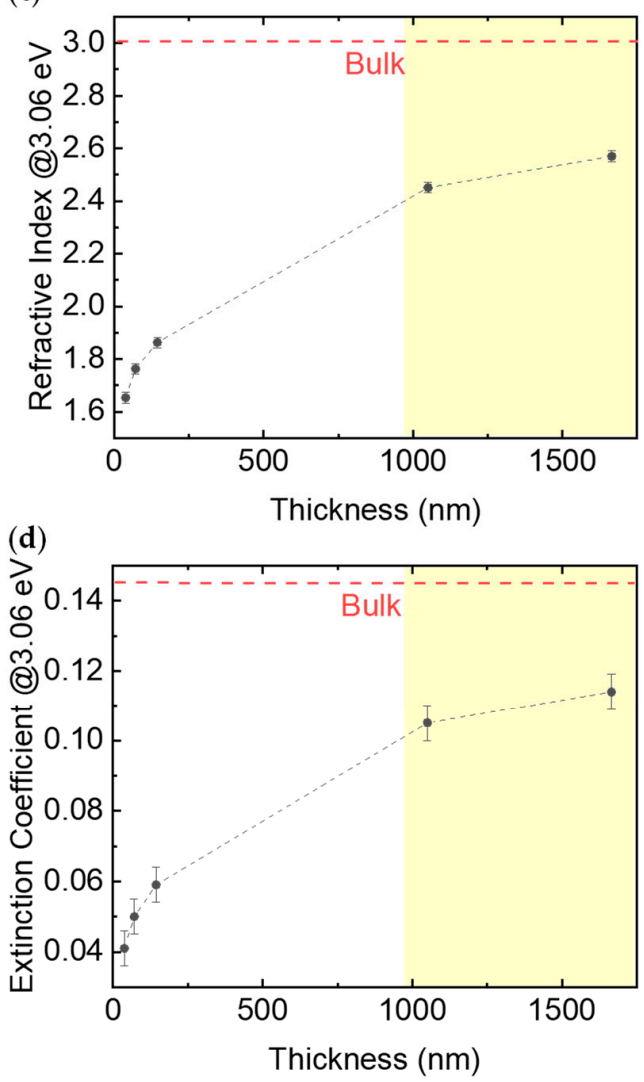

Figure 3. (a) Real $\left\langle\varepsilon_{1}\right\rangle$, and (b) imaginary, $\left\langle\varepsilon_{2}\right\rangle$, parts of the pseudodielectric function, $\langle\varepsilon\rangle=\left\langle\varepsilon_{1}\right\rangle+$ $i\left\langle\varepsilon_{2}\right\rangle$, of exfoliated GaS with different thicknesses. (c) Extinction coefficient and (d) refractive index at $3.06 \mathrm{eV}$ as a function of thickness. The reference values for the refractive index of bulk crystal GaS are also shown for comparison. The error bar on the refractive index and extinction coefficient comes from the errors on the GaS volume fraction percentage and voids volume fraction percentage of the BEMA approximation of the ellipsometric fitting.

\subsection{Electrical Characterization}

Figure $4 a$,b show the I-V characteristics of the thick $(1665 \mathrm{~nm})$ and thin $(38 \mathrm{~nm})$ GaS samples under dark, visible, and UV (405 nm) light irradiation. The dark current 
is in the range $10^{-13} \mathrm{~A}-10^{-15} \mathrm{~A}$ for the thick and thin samples, which is important for good detectivity in photodetectors. In the dark, the resistivity of the thick sample is $4.8 \times 10^{7} \Omega \cdot \mathrm{cm}$, in agreement with the literature [26,28,29]; it decreases to $8.2 \cdot 10^{4} \Omega \cdot \mathrm{cm}$ and $1.1 \cdot 10^{3} \Omega \cdot \mathrm{cm}$ upon illumination with visible and UV light, respectively. For the thin sample, the resistivity decreases from the dark value of $1.3 \cdot 10^{7} \Omega \cdot \mathrm{cm}$ to $7.4 \cdot 10^{6} \Omega \cdot \mathrm{cm}$ and $5.9 \cdot 10^{6} \Omega \cdot \mathrm{cm}$ upon visible and UV light irradiation, respectively. This is due to the lower light absorption indicated by the lower refractive index, i.e., polarizability and extinction coefficient (as shown in Figure 3). For samples thicker than $1000 \mathrm{~nm}$, the photocurrent, which increases by $4-5$ orders of magnitude, is linear with the applied voltage in the $-10 \mathrm{~V}$ to $+10 \mathrm{~V}$ range (see Figure $4 \mathrm{a}$ ), whereas a slight thermal displacement is seen for the thin sample (see Figure $4 b$ ).

(a)

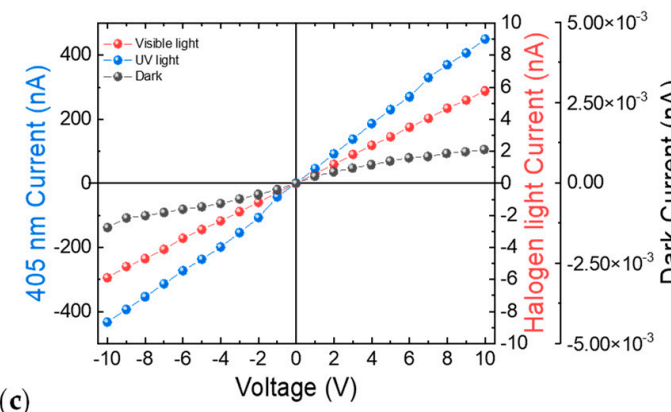

(c)

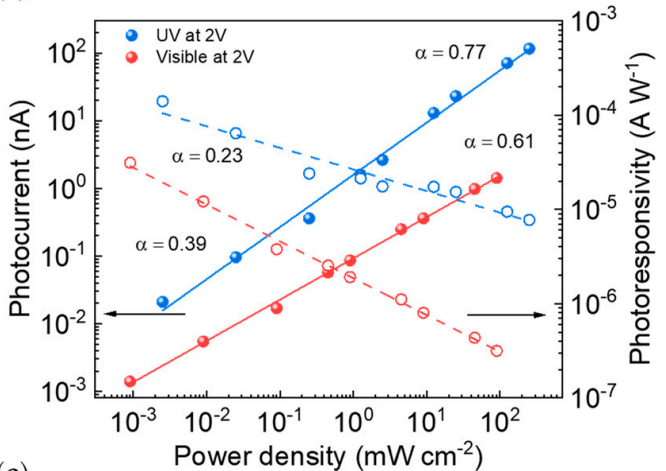

(e)

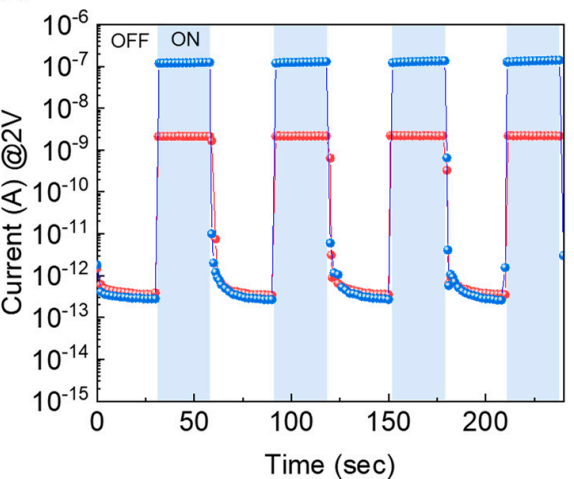

(b)

Thin $(38 \mathrm{~nm})$ sample

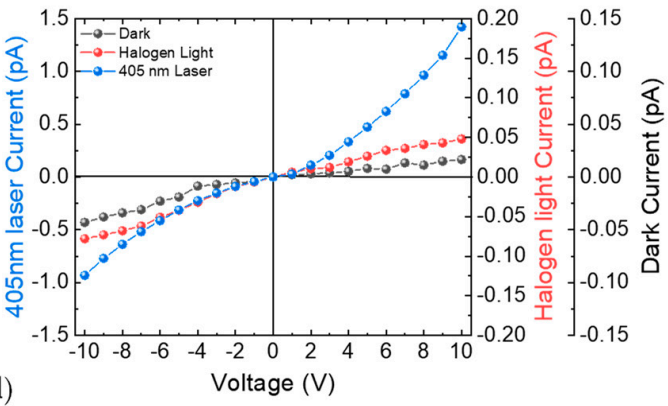

d)
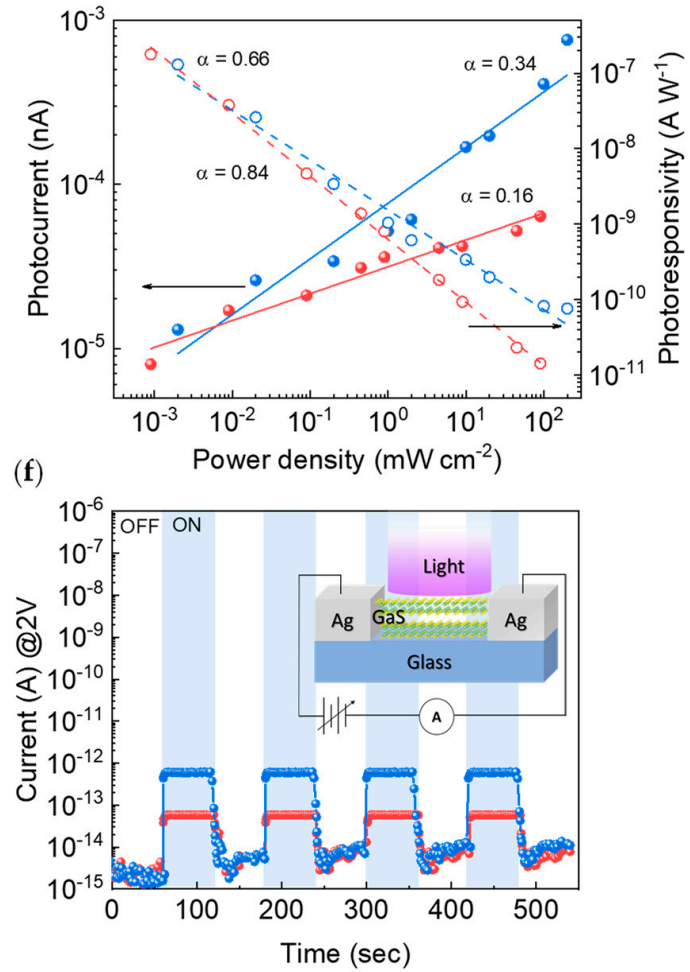

Figure 4. I-V characteristic of (a) thick and (b) thin exfoliated GaS samples in dark, visible light $\left(100 \mathrm{~mW} \mathrm{~cm}^{-2}\right)$, and UV-light $\left(250 \mathrm{~mW} \mathrm{~cm}^{-2}\right)$ irradiation. Photocurrent and photoresponsivity at $2 \mathrm{~V}$ as a function of visible and UV light power density for the (c) thick and (d) thin GaS samples; photocurrent versus time in response to light on/off under visible and UV light (the voltage is kept constant at $2 \mathrm{~V}$ ) for (e) thick and (f) thin GaS samples.

In order to gain further insights into the effect of the exfoliation process and thickness on the photoresponsivity, Figure $4 c-d$ show that the photocurrent increases with the 
incident optical power. The data plotted in a log-log scale show a linear trend consistently with the equation:

$$
I_{p h}=A \cdot P^{\alpha},
$$

where $A$ is a quantity related to the photoresponsivity and $\alpha$ is a dimensionless parameter containing information on the traps present in the system, $\alpha=1$ is expected in the case of a trap-free photodetector and decreases in the presence of traps. From Figure $4 c, \alpha=0.61$ and $\alpha=0.77$ are derived for the thick GaS, under visible and UV light, respectively. Conversely, thin GaS (see Figure $4 \mathrm{~d}$ ) has $\alpha=0.16$ and $\alpha=0.34$ for visible and UV light, respectively, which is consistent with the higher density of defects shown by SEM in Figure 1; a higher degree of oxidation, shown in Figure 2 by XPS; and higher voids percentage in the ellipsometry model, shown in Figure 3. A non-unity exponent of $0<\alpha<1$ in Equation (1) is often found in the low-dimensional photodetectors as a result of the complex process of carrier generation, trapping, and recombination within low-dimensional semiconductors with large surface-area-to-volume ratios, which is due to the long-lived states from the surface and interface traps [30]. As reported by Szałajko et al. [30], one of the possible processes involved in the nonlinear behavior of the photoresponsivity of $\mathrm{GaS}$ is the surface recombination, causing a decrease in the GaS velocity of carriers with the increase in illumination intensity.

The lower value of $\alpha$ obtained under visible light irradiation indicates the contributions of trap states below and within the bandgap originated by structural defects, while UV irradiation ( $405 \mathrm{~nm}$ is above the energy gap of $\mathrm{GaS}$ ) mainly involves band-to-band states. Trap states also influence the dynamics of the GaS photodetectors [31]. According to Equation (1), the photoresponsivity $(R)$, defined as

$$
R=\frac{I_{p h}}{P_{o p t}}
$$

decreases with the increase in the power density of light, following $R \sim P^{1-\alpha}$ as shown in Figure $4 c-d$, where $R$ is a parameter evaluating the sensitivity of the photoresponsive system, $I_{p h}$ is the photocurrent, and $P_{o p t}$ is the light power. If the photocurrent linearly depends on the light intensity, $R$ is a constant; conversely, in Figure $4 c-d, R$ decreases with the increase in incident power, which is typical for most photodetectors that use exciton separation. As the excitation power increases, more photoinduced electron-hole pairs are generated, and their interactions cause a loss of energy and efficiency during charge extraction [32]. Under high illumination intensities, the density of available states is reduced, resulting in a saturation of the photoresponsivity.

The photoresponsivity under UV light is $1.5 \cdot 10^{-4} \mathrm{~A} \cdot \mathrm{W}^{-1}$ when measured at $405 \mathrm{~nm}$ on centimeter-scale GaS compared with $2 \cdot 3 \cdot 10^{-4} \mathrm{~A} \cdot \mathrm{W}^{-1}$ when measured at $490 \mathrm{~nm}$ by $\mathrm{Hu}$ et al. [16], as well as with photoresponsivity of other bidimensional materials, as summarized in Table 1. The switching on/off of the photocurrent, i.e., the dynamic of the photo-to-dark current ratio (PDCR), or on/off ratio, defined as the ratio between the photocurrent $I_{p h}$ and the dark current $I_{\text {off }}$,

$$
\text { PDCR }=\frac{I_{p h}}{I_{o f f}}=\frac{I_{o n}-I_{o f f}}{I_{o f f}},
$$

is shown in Figure 4e-f, demonstrating a good GaS stability and reproducibility over several cycles; the sharp rising edge indicates an instant response. Noteworthy, the response time is less than $100 \mathrm{~ms}$ (our detection limit), which is two-to-three orders less than other bidimensional semiconductors (for comparison, a response time of $\mathrm{MoS}_{2}$ for UV light of $\approx 41 \mathrm{~s}$ has been reported) [20]. 
Table 1. Responsivity in typical low-dimensional photodetectors with experimental conditions.

\begin{tabular}{|c|c|c|c|c|}
\hline Material & $\begin{array}{c}\text { Responsivity } \\
\left(A \cdot W^{-1}\right)\end{array}$ & $\begin{array}{l}\text { Voltage } \\
\text { (V) }\end{array}$ & $\begin{array}{l}\text { Detection Range } \\
\text { Wavelength (nm) }\end{array}$ & Reference \\
\hline GaS (on glass) & $3.1 \cdot 10^{-5}$ & 2 & Visible & This work \\
\hline GaS (on glass) & $1.4 \cdot 10^{-4}$ & 2 & 405 & This work \\
\hline $\mathrm{GaS}$ (on $\mathrm{SiO}_{2} / \mathrm{Si}$ ) & $2.3 \cdot 10^{-4}$ & 2 & 490 & [14] \\
\hline $\mathrm{GaS}$ (on $\mathrm{SiO}_{2} / \mathrm{Si}$ ) & 4.7 & 20 & 275 & [22] \\
\hline $\mathrm{GaS}$ (on $\mathrm{SiO}_{2} / \mathrm{Si}$ ) & 1.9 & 20 & 275 & [32] \\
\hline Graphene & $5 \cdot 10^{-4}$ & & 1550 & [33] \\
\hline Graphene & 8.61 & & Visible-MIR & [34] \\
\hline $\mathrm{MoS}_{2}\left(\right.$ on $\left.\mathrm{SiO}_{2} / \mathrm{Si}\right)$ & $5 \cdot 10^{-4}$ & 1 & Visible & [35] \\
\hline $\mathrm{SnS}_{2}\left(\right.$ on $\left.\mathrm{SiO}_{2} / \mathrm{Si}\right)$ & $8.8 \cdot 10^{-3}$ & 2 & 457 & [36] \\
\hline
\end{tabular}

The PDCR relates to the optical and structural properties, as summarized in Figure 5. The PDCR increases with the thickness, with the extinction coefficient, and with the crystal quality of the samples. Specifically, the PDCR for the $1665 \mathrm{~nm}$ thick sample, calculated at $2 \mathrm{~V}$ bias voltage, is $6.7 \cdot 10^{3}$ and $3.7 \cdot 10^{5}$ under visible and UV light, respectively, whereas it is 40 (UV) and 3.5 (visible) for the $38 \mathrm{~nm}$ thin sample.

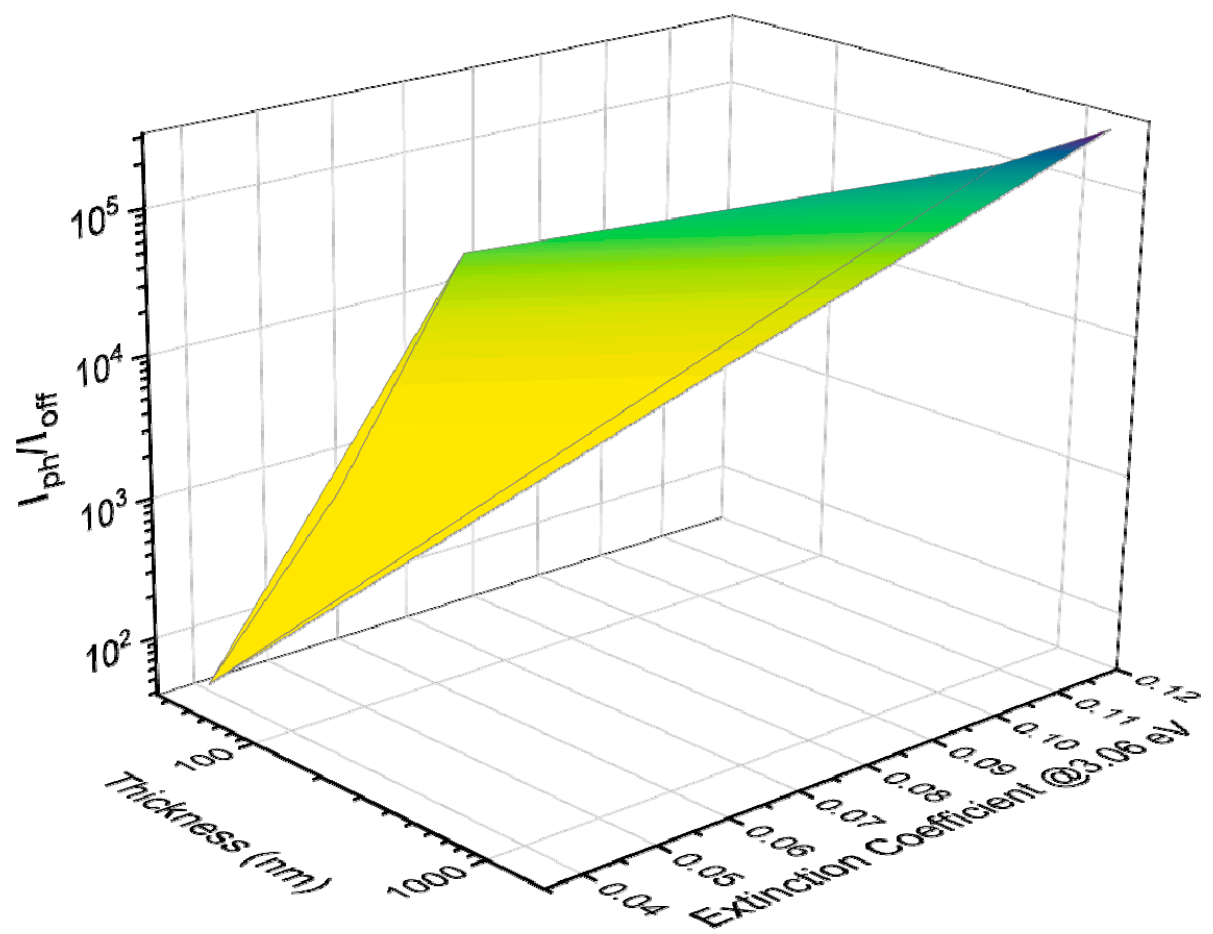

Figure 5. Photo-to-dark current ratio $I_{p h} / I_{\text {off }}$ under UV-light $(405 \mathrm{~nm})$ irradiation as a function of the thickness and of the extinction coefficient at $3.06 \mathrm{eV}$.

\section{Conclusions}

In summary, our results provided insights into the interplay between structure, defects, optical properties, and UV photoconductivity of centimeter-size exfoliated GaS samples of various thicknesses. Specifically, we showed that the GaS photoresponsivity increases with increases in the GaS thickness of exfoliated samples. GaS with a thickness $>1000 \mathrm{~nm}$ results in a UV photoresponsivity of $1.5 \cdot 10^{-4} \mathrm{AW}^{-1}$ with a photocurrent on/off ratio of $\sim 10^{5}$ stable over several on/off cycles and a quick response time of less than $100 \mathrm{~ms}$. As the thickness of the exfoliated GaS samples decreases with increases in exfoliation runs, the photoresponsivity also decreases due to defects introduced by the exfoliation process and a lower light absorption. 
These results are useful in designing a new generation of layered GaS UV photodetectors for medical diagnostics, ozone sensing, and optical communication systems.

Author Contributions: Conceptualization, S.D., Y.G., and M.L.; methodology, S.D. and Y.G.; validation, M.M.G., F.P., and G.V.B.; formal analysis, S.D. and G.V.B.; investigation, S.D., Y.G., M.M.G., F.P., and G.V.B.; resources, M.L.; data curation, S.D.; writing—original draft preparation, S.D.; writingreview and editing, S.D., Y.G., M.M.G., F.P., and G.V.B.; supervision, M.L.; project administration, M.L.; funding acquisition, M.L. All authors have read and agreed to the published version of the manuscript.

Funding: This research received funding from the European Union's Horizon 2020 research and innovation programme under grant agreement No 899598-PHEMTRONICS.

Institutional Review Board Statement: Not applicable.

Informed Consent Statement: Not applicable.

Data Availability Statement: The data in this study are available from the corresponding author upon request.

Acknowledgments: We acknowledge Giovanni Bruno for his helpful discussions during data analysis and our collaborator Sig. Alberto Sacchetti for assistance in performing the experiments. We also acknowledge Teresa Sibillano and Cinzia Giannini from Istituto di Cristallografia IC-CNR for providing the XRD measurements.

Conflicts of Interest: The authors declare no conflict of interest.

\section{References}

1. Cui, Y.; Peng, L.; Sun, L.; Qian, Q.; Huang, Y. Two-dimensional few-layer group-III metal monochalcogenides as effective photocatalysts for overall water splitting in the visible range. J. Mater. Chem. A 2018, 6, 22768-22777. [CrossRef]

2. Zhuang, H.L.; Hennig, R.G. Single-Layer Group-III Monochalcogenide Photocatalysts for Water Splitting. Chem. Mater. 2013, 25, 3232-3238. [CrossRef]

3. Late, D.J.; Liu, B.; Luo, J.; Yan, A.; Matte, H.S.S.R.; Grayson, M.; Rao, C.N.R.; Dravid, V.P. GaS and GaSe Ultrathin Layer Transistors. Adv. Mater. 2012, 24, 3549-3554. [CrossRef] [PubMed]

4. Buscema, M.; Island, J.O.; Groenendijk, D.J.; Blanter, S.I.; Steele, G.A.; van der Zant, H.S.J.; Castellanos-Gomez, A. Photocurrent generation with two-dimensional van der Waals semiconductors. Chem. Soc. Rev. 2015, 44, 3691-3718. [CrossRef] [PubMed]

5. Brebner, J.L. The optical absorption edge in layer structures. J. Phys. Chem. Solids 1964, 25, 1427-1433. [CrossRef]

6. Aulich, E.; Brebner, J.L.; Mooser, E. Indirect Energy Gap in GaSe and GaS. Phys. Status Solidi 1969, 31, 129-131. [CrossRef]

7. Cingolani, A.; Minafra, A.; Tantalo, P.; Paorici, C. Edge emission in GaSe and GaS. Phys. Status Solidi 1971, 4, K83-K85. [CrossRef]

8. Adachi, S.; Hamaguchi, C. Resonant Brillouin Scattering in GaSe and GaS. J. Phys. Soc. Jpn. 1980, 48, 1981-1989. [CrossRef]

9. Ho, C.H.; Lin, S.L. Optical properties of the interband transitions of layered gallium sulfide. J. Appl. Phys. 2006, 100, 083508. [CrossRef]

10. Tverjanovich, A.; Khomenko, M.; Bereznev, S.; Fontanari, D.; Sokolov, A.; Usuki, T.; Ohara, K.; Le Coq, D.; Masselin, P.; Bychkov, E. Glassy GaS: Transparent and unusually rigid thin films for visible to mid-IR memory applications. Phys. Chem. Chem. Phys. 2020, 22, 25560-25573. [CrossRef]

11. Carey, B.J.; Ou, J.Z.; Clark, R.M.; Berean, K.J.; Zavabeti, A.; Chesman, A.S.R.; Russo, S.P.; Lau, D.W.M.; Xu, Z.Q.; Bao, Q.; et al. Wafer-scale two-dimensional semiconductors from printed oxide skin of liquid metals. Nat. Commun. 2017, 8, 1-10. [CrossRef] [PubMed]

12. Jung, C.S.; Shojaei, F.; Park, K.; Oh, J.Y.; Im, H.S.; Jang, D.M.; Park, J.; Kang, H.S. Red-to-Ultraviolet Emission Tuning of Two-Dimensional Gallium Sulfide/Selenide. ACS Nano 2015, 9, 9585-9593. [CrossRef] [PubMed]

13. MacInnes, A.N.; Power, M.B.; Barron, A.R. Chemical vapor deposition of gallium sulfide: Phase control by molecular design. Chem. Mater. 1993, 5, 1344-1351. [CrossRef]

14. Wang, X.; Sheng, Y.; Chang, R.-J.; Lee, J.K.; Zhou, Y.; Li, S.; Chen, T.; Huang, H.; Porter, B.F.; Bhaskaran, H.; et al. Chemical Vapor Deposition Growth of Two-Dimensional Monolayer Gallium Sulfide Crystals Using Hydrogen Reduction of $\mathrm{Ga}_{2} \mathrm{~S}_{3}$. ACS Omega 2018, 3, 7897-7903. [CrossRef] [PubMed]

15. Eriguchi, K.; Biaou, C.; Das, S.; Yu, K.M.; Wu, J.; Dubon, O.D. Temperature-dependent growth of hexagonal and monoclinic gallium sulfide films by pulsed-laser deposition. AIP Adv. 2020, 10, 105215. [CrossRef]

16. Hu, P.; Wang, L.; Yoon, M.; Zhang, J.; Feng, W.; Wang, X.; Wen, Z.; Idrobo, J.C.; Miyamoto, Y.; Geohegan, D.B.; et al. Highly Responsive Ultrathin GaS Nanosheet Photodetectors on Rigid and Flexible Substrates. Nano Lett. 2013, 13, 1649-1654. [CrossRef] [PubMed] 
17. Harvey, A.; Backes, C.; Gholamvand, Z.; Hanlon, D.; McAteer, D.; Nerl, H.C.; McGuire, E.; Seral-Ascaso, A.; Ramasse, Q.M.; McEvoy, N.; et al. Preparation of Gallium Sulfide Nanosheets by Liquid Exfoliation and Their Application As Hydrogen Evolution Catalysts. Chem. Mater. 2015, 27, 3483-3493. [CrossRef]

18. Zhang, C.; Park, S.-H.; Ronan, O.; Harvey, A.; Seral-Ascaso, A.; Lin, Z.; McEvoy, N.; Boland, C.S.; Berner, N.C.; Duesberg, G.S.; et al. Enabling Flexible Heterostructures for Li-Ion Battery Anodes Based on Nanotube and Liquid-Phase Exfoliated 2D Gallium Chalcogenide Nanosheet Colloidal Solutions. Small 2017, 13, 1701677. [CrossRef] [PubMed]

19. Kato, K.; Umemura, N. Sellmeier equations for GaS and GaSe and their applications to the nonlinear optics in GaS $\mathrm{Se}_{1-\mathrm{x}}$. Opt. Lett. 2011, 36, 746-747. [CrossRef]

20. Opoku, F.; Akoto, O.; Asare-Donkor, N.K.; Adimado, A.A. Defect-engineered two-dimensional layered gallium sulphide molecular gas sensors with ultrahigh selectivity and sensitivity. Appl. Surf. Sci. 2021, 562, 150188. [CrossRef]

21. Yang, S.; Li, Y.; Wang, X.; Huo, N.; Xia, J.B.; Li, S.S.; Li, J. High performance few-layer GaS photodetector and its unique photo-response in different gas environments. Nanoscale 2014, 6, 2582-2587. [CrossRef] [PubMed]

22. Lu, Y.; Chen, J.; Chen, T.; Shu, Y.; Chang, R.J.; Sheng, Y.; Shautsova, V.; Mkhize, N.; Holdway, P.; Bhaskaran, H.; et al. Controlling Defects in Continuous 2D GaS Films for High-Performance Wavelength-Tunable UV-Discriminating Photodetectors. Adv. Mater. 2020, 32, 1906958. [CrossRef] [PubMed]

23. Gutierrez, Y.; Juan, D.; Dicorato, S.; Santos, G.; Duwe, M.; Thiese, P.H.; Giagregorio, M.M.; Palumbo, F.; Garcia-Fernnandez, P.; Junquera, J.; et al. Assessment of the Dielectric Function of Layered GaS from Bulk to Monolayer. Nanophotoics, 2022; submitted for publication.

24. Bruggeman, D.A.G. Berechnung verschiedener physikalischer Konstanten von heterogenen Substanzen. I. Dielektrizitätskonstanten und Leitfähigkeiten der Mischkörper aus isotropen Substanzen. Ann. Phys. 1935, 416, 636-664. [CrossRef]

25. Alencar, R.S.; Longuinhos, R.; Rabelo, C.; Miranda, H.; Viana, B.C.; Filho, A.G.S.; Cançado, L.G.; Jorio, A.; Ribeiro-Soares, J. Raman spectroscopy polarization dependence analysis in two-dimensional gallium sulfide. Phys. Rev. B 2020, 102, 165307. [CrossRef]

26. Gutiérrez, Y.; Giangregorio, M.M.; Dicorato, S.; Palumbo, F.; Losurdo, M. Exploring the Thickness-Dependence of the Properties of Layered Gallium Sulfide. Front. Chem. 2021, 9. [CrossRef]

27. Chakraborty, B.; Matte, H.S.S.R.; Sood, A.K.; Rao, C.N.R. Layer-dependent resonant Raman scattering of a few layer MoS2. J. Raman Spectrosc. 2013, 44, 92-96. [CrossRef]

28. Manfredotti, C.; Murri, R.; Rizzo, A.; Vasanelli, L. Hall effect in n-type GaS. Solid State Commun. 1976, 19, 339-342. [CrossRef]

29. Micocci, G.; Rella, R.; Siciliano, P.; Tepore, A. Investigation of electronic properties of gallium sulfide single crystals grown by iodine chemical transport. J. Appl. Phys. 1990, 68, 138-142. [CrossRef]

30. Szałajko, M.; Nowak, M. The influence of light intensity on surface recombination in GaS single crystals. Appl. Surf. Sci. 2007, 253, 3636-3641. [CrossRef]

31. Lopez-Sanchez, O.; Lembke, D.; Kayci, M.; Radenovic, A.; Kis, A. Ultrasensitive photodetectors based on monolayer MoS2. Nat Nanotechnol. 2013, 8, 497-501. [CrossRef] [PubMed]

32. Chen, T.; Lu, Y.; Sheng, Y.; Shu, Y.; Li, X.; Chang, R.-J.; Bhaskaran, H.; Warner, J.H. Ultrathin All-2D Lateral Graphene/GaS/Graphene UV Photodetectors by Direct CVD Growth. ACS Appl. Mater. Interfaces 2019, 11, 48172-48178. [CrossRef] [PubMed]

33. Xia, F.; Mueller, T.; Lin, Y.; Valdes-Garcia, A.; Avouris, P. Ultrafast graphene photodetector. Nat. Nanotechnol. 2009, 4, 839-843. [CrossRef] [PubMed]

34. Zhang, B.Y.; Liu, T.; Meng, B.; Li, X.; Liang, G.; Hu, X.; Wang, Q.J. Broadband high photoresponse from pure monolayer graphene photodetector. Nat. Commun. 2013, 4, 1811. [CrossRef] [PubMed]

35. Yin, Z.; Li, H.; Li, H.; Jiang, L.; Shi, Y.; Sun, Y.; Lu, G.; Zhang, Q.; Chen, X.; Zhang, H. Single-Layer MoS2 Phototransistors. ACS Nano 2012, 6, 74-80. [CrossRef]

36. Su, G.; Hadjiev, V.G.; Loya, P.E.; Zhang, J.; Lei, S.; Maharjan, S.; Dong, P.M.; Ajayan, P.; Lou, J.; Peng, H. Chemical Vapor Deposition of Thin Crystals of Layered Semiconductor SnS2 for Fast Photodetection Application. Nano Lett. 2015, 15, 506-513. [CrossRef] 